\title{
Skin Sensitivity to Aeroallergens in Allergic Rhinitis
}

\author{
Meenakshi Mishra, Arunabha Chakravarti, Raj Kumar
}

\section{ABSTRACT}

Background: Allergic rhinitis is a common IgE-mediated inflammatory condition of the nasal mucosa. Skin prick test (SPT) remains the most useful test for detecting specific allergic triggers associated with allergic rhinitis.

Objective: Our objective was to study the sensitivity to common aeroallergens in allergic rhinitis patients using SPT.

Materials and methods: We conducted SPT to 65 common aeroallergens on 100 patients more than 12 years of age with symptoms suggestive of allergic rhinitis.

Results: Sixty-one patients $(61 \%)$ tested positive for at least one aeroallergen, of which, 54 patients $(88.52 \%)$ showed polysensitization while seven patients $(11.48 \%)$ showed monosensitization. The most common aeroallergen group implicated in SPT positivity was insects (48\%); housefly (34\%) being the most commonly implicated insect.

Conclusion: This study highlights a high prevalence of sensitivity to aeroallergens in the Indian scenario. It stresses upon the fact that environmental control measures might still prove to be the mainstay of treatment in our scenario, owing to housefly being the most commonly implicated aeroallergen.

Keywords: Allergic rhinitis, Aeroallergens, Environmental control, Immunotherapy.

How to cite this article: Mishra M, Chakravarti A, Kumar R. Skin Sensitivity to Aeroallergens in Allergic Rhinitis. Clin Rhinol An Int J 2013;6(2):64-66.

Source of support: Nil

Conflict of interest: None declared

\section{INTRODUCTION}

Allergic rhinitis is a common inflammatory condition of the nasal mucosa characterized by sneezing, rhinorrhea, nasal congestion and pruritus. It is mediated by an IgEassociated response to indoor and outdoor environmental allergens. The prevalence of allergic rhinitis has been estimated to be between 15 and $20 \% .^{1}$ The challenges in diagnosing and evaluating allergic rhinitis continue. Specific $\mathrm{IgE}$ antibodies can be demonstrated by immediate reaction prick and intradermal skin tests, or by in vitro methods like radioallergosorbent test (RAST) and enzyme-linked immunosorbent assay (ELISA). ${ }^{2,3}$ Appropriate clinical evaluation and an accurate diagnosis using skin testing or serum IgE testing is needed to develop a holistic approach for treatment. Skin prick test (SPT) is the preferred diagnostic test because it is safer, less painful and more specific than the intracutaneous test, and cheaper and more sensitive than the RAST. ${ }^{2}$

The present study will help in identifying common aeroallergens implicated in causation of allergic rhinitis in the Indian setup. The avoidance of allergen will lead to improvement of patient's condition. It will also be useful in initiation of allergen-specific immunotherapy in patients whose symptoms are not controlled with environmental change and pharmacotherapy.

\section{MATERIALS AND METHODS}

This prospective, observational study was conducted on 100 patients aged 12 years or above with symptoms suggestive of allergic rhinitis attending the Department of Otorhinolaryngology and Head and Neck Surgery, Lady Hardinge Medical College and associated hospitals. The protocol for this study was approved by the hospital ethics committee, and all patients gave their informed consent. Patients with skin hyper-reactivity, generalized skin disease and prior history of anaphylaxis were excluded from the study.

$\mathrm{SPT}^{4}$ was performed against 65 common aeroallergens $(1: 10 \mathrm{w} / \mathrm{v}, 50 \%$ glycerinated) which included grasses (Cenchrus, Cynodon, Imperata, Pennisetum, Sorghum), weeds (Adhatoda, Ageratum, Amaranthus spinosus, Argemone, Artemisia, Asphodelus, Brassica, Cannabis, Cassia occidentalis, Chenopodium album, Chenopodium M, Dodonaea, Gynandropsis, Parthenium, Suaeda, Xanthium), trees (Cassia Siamea, Ehretia, Eucalyptus, Kigelia, Melia, Morus, Prosopis, Putranjiva, Ricinus, Salvadora, Holoptelea), dust (house dust, wheat dust, paper dust, cotton dust, house dust mite), fungi (Alternaria, Aspergillus fumigatus, Aspergillus tamarii, Candida, Cladosporium, Helminthosporium, Mucor, Phoma, Trichoderma, Rhizopus, Epicoccum), insects (cockroach male, cockroach female, housefly, rice weevil, mosquito, moth) and others (kapok cotton, wool, silk). The test was carried out in the Department of Respiratory Allergy and Clinical Immunology at VP Chest Institute, University of Delhi.

The patients who underwent this test were instructed not to take antihistaminics 7 days prior to the test and cough syrups 4 days prior to the test. The patients were however allowed to take inhaled medications. The positive control used was histamine and the negative control was buffered normal saline. The pricks were given on the ventral aspect of the forearm by a fine sharp needle, using separate needle for each prick, making sure that prick did not draw blood (Fig. 1). The mean diameter was calculated after 15 to 20 minutes as $(D+d) / 2$ where ' $D$ ' is the largest diameter and ' $d$ ' is the orthogonal or perpendicular diameter at the 


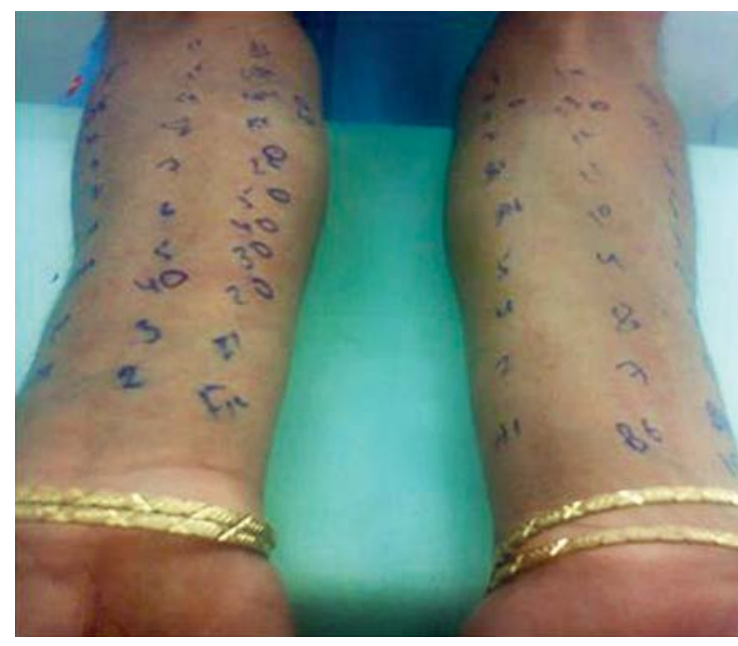

Fig. 1: Skin prick test

largest width of ' $D$. A positive result to a specific allergen was indicated by a mean wheal diameter measuring $3 \mathrm{~mm}$ or more than negative control.

\section{RESULTS}

Majority of patients were in the age group 20 to 30 years $(43 \%)$, with mean age of 27.49 years. The minimum age of presentation was 13 years and the maximum was 55 years. The study included 51 males (51\%) and 49 females (49\%). Family history of allergic conditions was found to be positive in 37 patients (37\%). Among the patients with positive family history, 27 patients $(72.97 \%)$ had a maternal family history and 10 patients $(27.03 \%)$ had a paternal family history.

Grading of the severity as per the Wilson et al scoring system revealed that 74 patients $(74 \%)$ had moderate rhinitis, 22 patients $(22 \%)$ had severe rhinitis and only four patients (4\%) had mild rhinitis. Sixty-one patients (61\%) tested positive for at least one aeroallergen; out of which seven patients $(11.48 \%)$ showed monosensitization and 54 patients $(88.52 \%)$ showed polysensitization.

The most common aeroallergen group implicated in SPT positivity was insects $(48 \%)$. The prevalence of other allergens were weeds $(29 \%)$, dust $(26 \%)$, trees $(23 \%)$, grass (13\%), fungi (12\%) and others (5\%) (Graph 1). The prevalence of individual aeroallergens is depicted in Graph 2. The most common insect implicated in causation of allergic rhinitis in our set-up was housefly, found positive in 34 patients $(34 \%)$ of the cases.

\section{DISCUSSION}

Allergic rhinitis is a global health problem due to its impact on quality of life and work productivity. The present study aimed to evaluate the clinical profile and sensitivity to

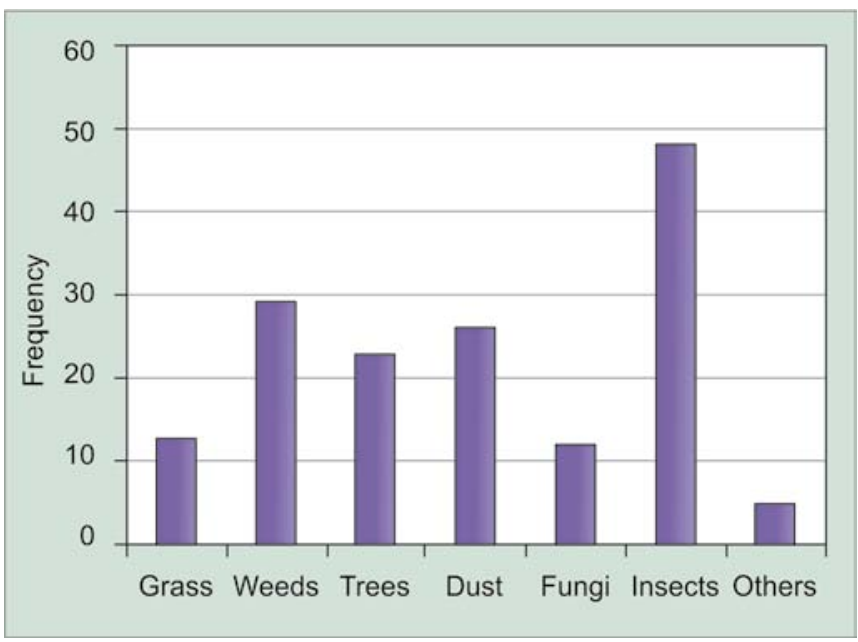

Graph 1: Prevalence of aeroallergen positivity

common aeroallergens in patients with allergic rhinitis in the Indian scenario. Review reveals a similar study conducted by Haahtela et al from Finland, in the year 1981 in which he evaluated the relationship between serum $\operatorname{IgE}$ and SPT using 6 common aeroallergens. Out of the 137 allergic rhinitis patients included in the study, $48 \%$ boys and $47 \%$ girls showed positivity to at least one allergen on SPT. ${ }^{5}$ A study conducted by Ahmed et al found $68.6 \%$ of the allergic patients as SPT positive. ${ }^{6}$ Aburuz et al in the year 2011 carried out SPT with 18 standardized allergen extracts in allergic rhinitis patients. Grasses mix (51.4\%), thistle weed $(46.9 \%)$ and olive tree $(45.3 \%)$ pollens were the most common seasonal allergens. Cat allergen was the most common perennial allergen (41.6\%), followed by dust mite $(32.9 \%)^{7}$

On Medline search, we did not come across studies with SPT being conducted on allergic rhinitis patients with such a large number (65) of aeroallergen extracts. This study highlights a high prevalence of sensitivity to aeroallergens in the Indian scenario. It brings to light some important aspects which may provide a breakthrough in the management of allergic rhinitis in our scenario.

The allergen group most commonly implicated was insects, more specifically housefly, thus stressing upon the fact that environmental control measures can play an important role in management of allergic rhinitis. It is advocated that if facilities and infrastructure exist, SPT should be performed in all patients with allergic rhinitis, so as to educate the patient about avoidance of the implicated aeroallergen.

\section{REFERENCES}

1. Greiner AN. Allergic rhinitis: impact of the disease and considerations for management. Med Clin North Am 2006 Jan;90(1):17-38. 
2. Badhwar AK, Druse HM. Allergic rhinitis. Med Clin North Am 1992 Jul;76(4):789-803.

3. Ferguson AC. RAST and allergic disease. B C Med J 1983;25:568-570.

4. Dreborg S. Skin testing. The safety of skin tests and the information obtained from using different methods and concentration of allergen. Allergy 1993 Oct;48(7):473-475.

5. Haahtela T, Jaakonmaki I. Relationship of allergen-specific IgE antibodies, skin prick tests and allergic disorders in unselected adolescents. Allergy 1981 May;36(4):251-256.

6. Ahmed A, Minhas K, Micheal S, Ahmad F. Prevalence of skin test reactivity to aeroallergens in the Pakistani population. Public Health 2011 May;125(5):324-326.

7. Aburuz S, Bulatova N, Tawalbeh M. Skin prick test reactivity to aeroallergens in Jordanian allergic rhinitis patients. East Mediterr Health J 2011 Jul;17(7):604-610.

\section{ABOUT THE AUTHORS}

\section{Meenakshi Mishra}

Junior Resident, Department of Otorhinolaryngology, Head and Neck Surgery, Lady Hardinge Medical College, New Delhi, India

\section{Arunabha Chakravarti (Corresponding Author)}

Professor, Department of Otorhinolaryngology, Head and Neck Surgery, Lady Hardinge Medical College, New Delhi, India, Phone: 09868093035, e-mail: drachakravarti@yahoo.co.in

\section{Raj Kumar}

Professor and Head, Department of Respiratory, Allergy and Applied Immunology, Vallabhbhai Patel Chest Institute, New Delhi, India 